FERMILAB-FN-0951-APC

\title{
Local Longitudinal Microwave Instability Limits During Bunch Rotation
}

\author{
K.Y. Ng \\ Fermi National Accelerator Laboratory, Batavia, IL 60510
}

(October 23, 2012)

\begin{abstract}
Bunch width compression can be accomplished by rf rotating an elongated bunch with minimal energy spread. The formation of tails at the two bunch ends produces disconnected regions in many time-advance slices. The stability limits of longitudinal microwave growth for each time-advance slice of the beam will be affected. This effect is studied and the Keil-Schnell stability limits for such time-advance slices are derived. Application is made to the bunch-width compression in the Fermilab Compressor Ring, destined for pion and subsequently muon production.
\end{abstract}




\section{Introduction}

The proton source for the muon collider or neutral factory at Fermilab consists of accumulating four bunches each of intensity $N_{b}=0.525 \times 10^{14}$ in the Accumulator (AC) using $2.5-\mathrm{MHz}$ rf. They are compressed to a final rms fractional energy spread of $\sigma_{E}=5.2 \times 10^{-4}$ and rms bunch length $\sigma_{\tau}=29.2 \mathrm{~ns}$ by lowering the rf voltage adiabatically. These four bunches are then transferred to the Compressor Ring (CR) one at a time for the neutrino factory or all at the same time for the muon collider. Inside the $\mathrm{CR}$, these bunches are rotated by the CR rf with a voltage of $240 \mathrm{kV}$ so as to create bunches as narrow as rms width $\sigma_{\tau}=3.2 \mathrm{~ns}$. The question to answer here is whether the bunches will develop longitudinal microwave instabilities during the bunch rotation.

Section 2 deals with rotation with a linear rf. The result is that the beam will become more stable against longitudinal microwave instability than its initial configuration as the rotation proceeds. At each moment of the rotation, the center time-advance slice has the most stringent stability limit.

Section 3 answers the same question when the nonlinearity of the rf waveform is taken into account. Because of the tight rf bucket, tails develop at the two ends of the bunch. This affects the energy distribution of the beam and therefore the stability limits. It turns out, however, that the beam is still more stable against longitudinal microwave instability than its initial configuration.

\section{Linear RF}

The Boussard-modified Keil-Schnell criterion [1, 2] for longitudinal microwave instability is

$$
\left|\frac{Z_{0}^{\|}}{n}\right| \lesssim \frac{2 \pi \eta E \sigma_{E}^{2}}{e I_{\mathrm{pk}} \beta^{2}}
$$

where $Z_{0}^{\|} / n$ is the coupling longitudinal impedance of the accelerator ring at revolution harmonic $n, E$ is the nominal energy of the beam particles, $\beta$ is the nominal velocity of the beam particles with respect to the velocity of light, $\eta$ is the slip parameter, and $I_{\mathrm{pk}}$ is the local peak current of the beam. In above, Gaussian distribution of the energy spread of the beam has been assumed. If we assume also Gaussian distribution of the bunch in linear 
distribution, [3] local peak current of the beam depends on the rms bunch length $\sigma_{\tau}$ as

$$
I_{\mathrm{pk}}=\frac{e N_{b}}{\sqrt{2 \pi} \sigma_{\tau}}
$$

where $N_{b}$ is the number of protons in the bunch. The Boussard-modified Keil-Schnell criterion can then be rewritten as

$$
\left|\frac{Z_{0}^{\|}}{n}\right| \lesssim \frac{(2 \pi)^{3 / 2} \eta E \sigma_{\tau} \sigma_{E}^{2}}{e N_{b} \beta^{2}} .
$$

During bunch rotation the bunch area, which is proportional to $\sigma_{\tau} \sigma_{E}$, remains constant. The bunch is therefore more stable against longitudinal microwave instability as the energy spread increases. In other words, the bunch is most susceptible to longitudinal microwave instability before the rf rotation when its fractional energy spread is compressed adiabatically to the minimum value of $\sigma_{E}=5.2 \times 10^{-4}$. It will becomes more stable as soon as the $\mathrm{rf}$ rotation raises its energy spread and squeezes its longitudinal width. To demonstrate this more clearly, we rotate rotate a bunch by a linear rf waveform and determine the instability limit stated in Eq. (2.1) at any instance of the rotation.

\subsection{Linear distribution and energy spread during rotation}

Start with a bunch with bi-Gaussian distribution in the longitudinal phase space. The rf rotates the bunch by $90^{\circ}$. We wish to compute the local linear density and its corresponding energy spread at each moment of the rotation.

To simplify the derivation, the energy coordinate $y$ is first scaled to the time coordinate $x$, so that the tips of the bunch rotate in a circle (instead of an ellipse) in the longitudinal phase space. The initial phase space distribution is

$$
f_{0}(x, y)=\frac{1}{2 \pi a b} \exp \left[-\frac{x^{2}}{2 a^{2}}-\frac{y^{2}}{2 b^{2}}\right]
$$

where $a=\sigma_{\tau 0}$, the initial rms time spread and $b$ is proportional to the initial rms energy spread. For simplicity we just identify $b=\sigma_{E 0}$. The rf rotation is just the rotation of the $x-y$ coordinates by the angle $\theta$. This is accomplished by the substitution,

$$
\left(\begin{array}{l}
x \\
y
\end{array}\right) \rightarrow\left(\begin{array}{rr}
\cos \theta & -\sin \theta \\
\sin \theta & \cos \theta
\end{array}\right)\left(\begin{array}{l}
x \\
y
\end{array}\right) .
$$


At the rotation angle $\theta$, the phase-space distribution is therefore

$$
\begin{aligned}
f_{\theta}(x, y)=\frac{1}{2 \pi a b} \exp [ & -x^{2} \frac{b^{2} \cos ^{2} \theta+a^{2} \sin ^{2} \theta}{2 a^{2} b^{2}}-y^{2} \frac{b^{2} \sin ^{2} \theta+a^{2} \cos ^{2} \theta}{2 a^{2} b^{2}} \\
& \left.-2 x y \frac{\left(a^{2}-b^{2}\right) \sin \theta \cos \theta}{2 a^{2} b^{2}}\right] .
\end{aligned}
$$

The linear distribution is obtained by integrating over $y$. We perform the integration

$$
\begin{aligned}
I & =\int d y \exp \left[-y^{2} \frac{b^{2} \sin ^{2} \theta+a^{2} \cos ^{2} \theta}{2 a^{2} b^{2}}-2 x y \frac{\left(a^{2}-b^{2}\right) \sin \theta \cos \theta}{2 a^{2} b^{2}}\right] \\
& =\int d y \exp \left[-\frac{y^{2}}{2 p^{2}}-2 q x y\right] \\
& =\int d y \exp \left[-\frac{\left(y+2 p^{2} q x\right)^{2}}{2 p^{2}}+2 p^{2} q^{2} x^{2}\right]=\sqrt{2 \pi} p e^{2 p^{2} q^{2} x^{2}},
\end{aligned}
$$

where

$$
p^{2}=\frac{a^{2} b^{2}}{b^{2} \sin ^{2} \theta+a^{2} \cos ^{2} \theta} \quad \text { and } \quad q=\frac{\left(a^{2}-b^{2}\right) \sin \theta \cos \theta}{2 a^{2} b^{2}} .
$$

It is obvious that $p$ is the rms energy spread at $x=0$, or the center time-advance slice of the bunch. The linear distribution is

$$
\lambda_{\theta}(x)=\frac{\sqrt{2 \pi} p}{2 \pi a b} \exp \left[-\frac{x^{2}}{2 a^{2} b^{2}}\left(b^{2} \cos ^{2} \theta+a^{2} \sin ^{2} \theta\right)+2 p^{2} q^{2} x^{2}\right] .
$$

The exponent becomes

$$
\begin{aligned}
{[] } & =-\frac{x^{2}}{2 a^{2} b^{2}}\left[\left(b^{2} \cos ^{2} \theta+a^{2} \sin ^{2} \theta\right)-4 a^{2} b^{2} p^{2} q\right] \\
& =-\frac{x^{2}}{2 a^{2} b^{2}}\left[\left(b^{2} \cos ^{2} \theta+a^{2} \sin ^{2} \theta\right)-4 a^{2} b^{2} \frac{a^{2} b^{2}}{b^{2} \sin ^{2} \theta+a^{2} \cos ^{2} \theta} \frac{\left(a^{2}-b^{2}\right)^{2} \sin ^{2} \theta \cos ^{2} \theta}{4 a^{4} b^{4}}\right] \\
& =-\frac{x^{2}\left[\left(b^{4}+a^{4}\right) \sin ^{2} \theta \cos ^{2} \theta+a^{2} b^{2}\left(\sin ^{4} \theta+\cos ^{4} \theta\right)-\left(a^{2}-b^{2}\right)^{2} \sin ^{2} \theta \cos ^{2} \theta\right]}{2 a^{2} b^{2}\left(b^{2} \sin ^{2} \theta+a^{2} \cos ^{2} \theta\right)} \\
& =-\frac{x^{2}}{2\left(b^{2} \sin ^{2} \theta+a^{2} \cos ^{2} \theta\right)} .
\end{aligned}
$$

Or the linear distribution at angle $\theta$ is

$$
\lambda_{\theta}(x)=\frac{1}{\sqrt{2 \pi} \sigma_{\tau \theta}} e^{-x^{2} / 2 \sigma_{\tau \theta}^{2}},
$$

with the rms time spread of

$$
\sigma_{\tau \theta}=\sqrt{b^{2} \sin ^{2} \theta+a^{2} \cos ^{2} \theta}
$$


which reduces to $a$ and $b$, respectively, at $\theta=0$ and $\pi / 2$, as expected.

The rms energy spread is obtained by taking the second moment of the phase-space distribution in the $y$-direction, or

$$
\sigma_{E \theta}^{2}(x)=\frac{\int d y y^{2} f_{\theta}(x, y)}{\int d y f_{\theta}(x, y)}
$$

With $\tilde{y}=y+2 p^{2} q x$, this becomes

$$
\begin{aligned}
\sigma_{E \theta}^{2}(x) & =\frac{\int d \tilde{y}\left(\tilde{y}-2 p^{2} q x\right)^{2} e^{-\tilde{y}^{2} / 2 p^{2}+2 p^{2} q^{2} x^{2}}}{\int d \tilde{y} e^{-\tilde{y}^{2} / 2 p^{2}+2 p^{2} q^{2} x^{2}}} \\
& =\frac{1}{\sqrt{2 \pi} p} \int d \tilde{y}\left(\tilde{y}^{2}+4 p^{4} q^{2} x^{2}\right) e^{-\tilde{y}^{2} / 2 p^{2}} \\
& =p^{2}\left[1+4 p^{2} q^{2} x^{2}\right] \\
& =\frac{a^{2} b^{2}}{b^{2} \sin ^{2} \theta+a^{2} \cos ^{2} \theta}\left[1+\frac{\left(a^{2}-b^{2}\right)^{2} \sin ^{2} \theta \cos ^{2} \theta}{b^{2} \sin ^{2} \theta+a^{2} \cos ^{2} \theta} x^{2}\right] .
\end{aligned}
$$

\subsection{Keil-Schnell Criterion}

Keil-Schnell criterion for microwave stability is

$$
\left|\frac{Z_{0}^{\|}}{n}\right| \leq \frac{2 \pi|\eta| E \sigma_{E \theta}^{2}}{e N_{b} \lambda_{\theta}}
$$

In other words, the stability limit is determined by the ratio

$$
\frac{\sigma_{E \theta}^{2}}{\lambda_{\theta}}=\sqrt{2 \pi} \sigma_{\tau \theta} p^{2}\left[1+4 p^{2} q^{2} x^{2}\right] e^{x^{2} / 2 \sigma_{\tau \theta}^{2}} .
$$

We find that, for any $\theta$, the rms energy spread is the smallest and the linear density is the highest when $x=0$. It is clear that the stability limit will be first reached when $x=0$ or at the center of the bunch, and this is true at any stage of the bunch rotation. In other words, during the bunch rotation by the rf, we need to pay attention only at the center of the bunch or only at $x=0$. Keil-Schnell criterion for microwave stability for the rotating bunch then becomes

$$
\left|\frac{Z_{0}^{\|}}{n}\right| \leq \frac{(2 \pi)^{3 / 2}|\eta| E}{e N_{b}} \sigma_{\tau \theta} p^{2}=\frac{(2 \pi)^{3 / 2}|\eta| E}{e N_{b}} \frac{a^{2} b^{2}}{\sqrt{b^{2} \sin ^{2} \theta+a^{2} \cos ^{2} \theta}}
$$


The above can also be rewritten as

$$
\left|\frac{Z_{0}^{\|}}{n}\right| \leq\left|\frac{Z_{0}^{\|}}{n}\right|_{\theta=0} \frac{1}{\sqrt{\frac{b^{2}}{a^{2}} \sin ^{2} \theta+\cos ^{2} \theta}} .
$$

Since $b / a \ll 1$, it is obvious the stability increases as $\theta$ increases. The last factor equals 1 at $\theta=0$ and increases to $a / b$ when $\theta=\pi / 2$.

\section{Rotation with Nonlinear RF Waveform}

The rf waveform is sinusoidal and therefore nonlinear. The ends of the bunch will be rotated at a much slower rate than the bunch center, resulting in tail formation at the two ends of the rotated bunch. A simulation is performed starting with a bi-Gaussian distributed bunch of rms time advance spread $\sigma_{\tau 0}=29.2 \mathrm{~ns}$ and rms fraction energy spread $\sigma_{E 0}=5.2 \times 10^{-4}$ in the CR, displayed as black dots in Fig. 1. The bunch configuration after 640 turns is shown in red. The small-amplitude synchrotron tune is $\nu_{s}=4.16 \times 10^{-4}$ and the corresponding period is $T_{s}=2403$ turns. Because of the formation of the tails at the ends of the bunch, the energy spread is less than what we expect in a linear rf. We select all particles between time advance $\pm 1 \mathrm{~ns}$. There are 29352 particles with $\mathrm{rms}$ energy spread $\sigma_{E}=5.73 \times 10^{-3}$, out of a total of a hundred thousand macroparticles employed in the simulation. If the rf waveform were linear, the energy spread after a $\pi / 2$ rotation would have been

$$
\sigma_{E}=\frac{\beta^{2} \nu_{s} f_{0} \sigma_{\tau 0}}{|\eta|}=7.37 \times 10^{-3},
$$

where $f_{0}=0.967 \mathrm{MHz}$ is the $\mathrm{CR}$ nominal revolution frequency and the momentum compaction factor $\alpha=0.001$ has been used. We also try to sample particles between $\pm 0.7 \mathrm{~ns}$. There are 19168 macroparticles in this 0-ns time-advance slice. The rms fractional energy spread is $5.71 \times 10^{-3}$, not much different from the sampling of \pm 1 ns. The Boussard-modified Keil-Schnell criterion of Eq. (2.1) can be interpreted as

$$
\left|\frac{Z_{0}^{\|}}{n}\right| \sim \frac{\sigma_{E}}{\text { particle density }} .
$$

Liouville theorem tells us that the local particle density in the longitudinal phase space remains unchanged for the core of the bunch. Thus the stability limit is proportional to the energy spread. The above analysis says that the stability limit provided by a realistic 


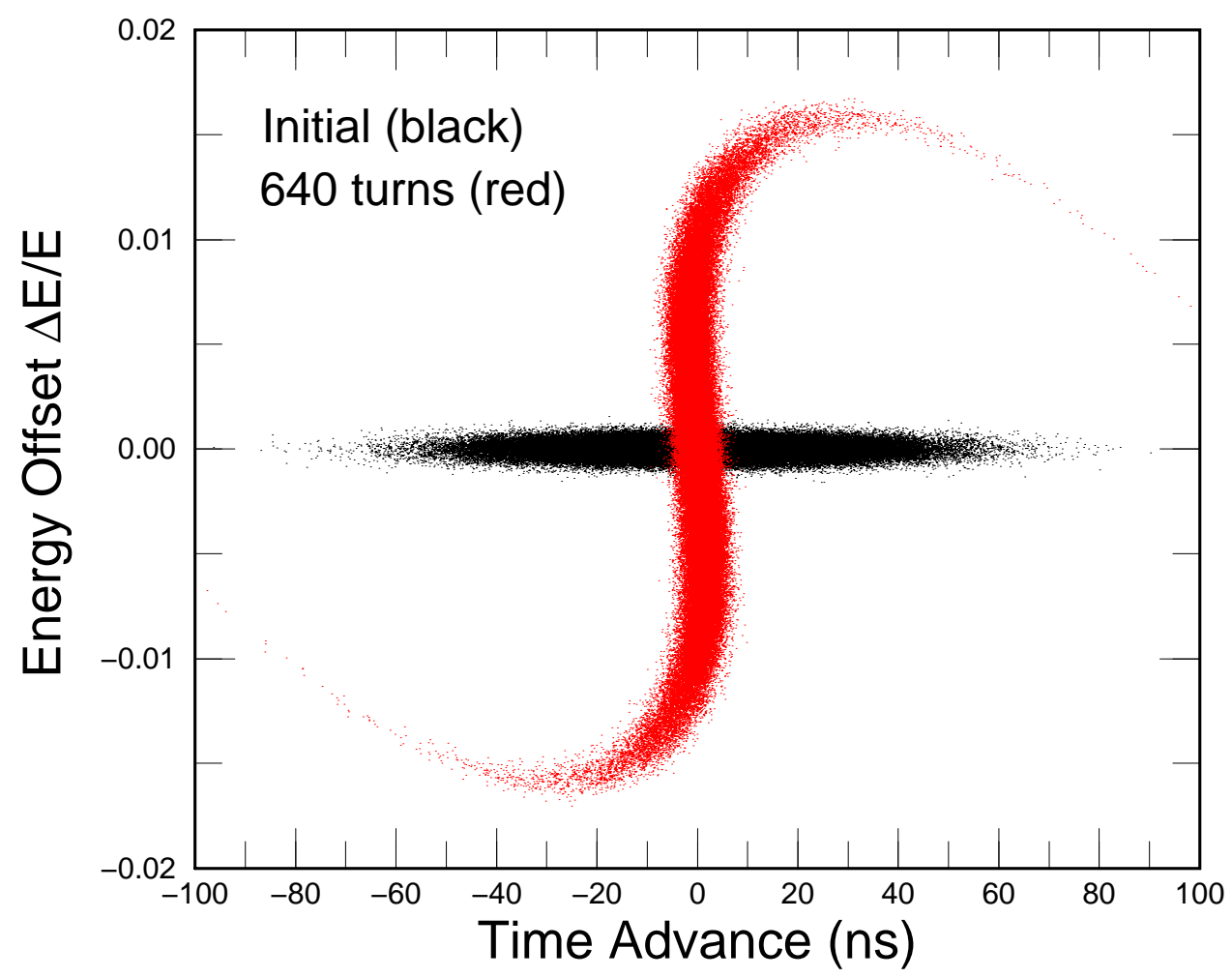

Figure 1: (Color) A bunch in the CR initially bi-Gaussian distributed with $\sigma_{\tau 0}=29.2 \mathrm{~ns}$ and $\sigma_{E 0}=5.2 \times 10^{-4}$ is shown in black dots. It is rotated by a $2.5-\mathrm{MHz}$ rf of peak voltage $240 \mathrm{kV}$. Red dots show the bunch rotation after 640 turns. Small-amplitude synchrotron period is 2403 turns. .

sinusoidal rf waveform would be smaller than that provided by a linear $\mathrm{rf}$ waveform, because of the smaller energy spread of the center slice after rotation. Nevertheless, this limit is much larger than that before the bunch rotation. Numerically, we find the local current at the 0 -ns time-advance slice to be $I_{\mathrm{pk}}=1155 \mathrm{~A}$, and the Boussard-modified Keil-Schnell stability limit is

$$
\left|\frac{Z_{0}^{\|}}{n}\right| \lesssim 16.9 \Omega
$$

The rms bunch length during the rotation is shown in Fig. 2. The black curve is the bunch rms width $\mathrm{w}$ hen the whole bunch including the end tails is taken into account. The minimum is $\sigma_{\tau}=4.89 \mathrm{~ns}$. However, if the tails are excluded, the beam width is much narrower. The red curve includes only beam particles with fractional energy offset within \pm 0.0025 . The minimum is $\sigma_{\tau}=2.29 \mathrm{~ns}$ at turn 639 . Actually the width of this energy-offset slice does not change much from turn 550 to turn 675 . 




Figure 2: (Color) Rms bunch length during the rf rotation. Black: The whole bunch including tails is taken into account. Red: Only an energy-offset slice \pm 0.0025 is included in the rms bunch length computation .

\section{$3.1 \quad$ Overlapped beams}

The purpose of $\mathrm{rf}$ bunch rotation is to obtain a bunch of short duration to hit the target in the generation of pions and subsequently muons. Because of the development of tails by the sinusoidal $\mathrm{rf}$ waveform, sometimes one may like to rotate a little more $\pi / 2$ so that more beam particles will be included in the later truncated narrow bunch. Figure 3 shows the result of the rotation after 660 turns. The longitudinal microwave stability limit for the 0 -ns time-advance slice remains as $\left|Z_{0}^{\|} / n\right| \lesssim 16.9 \Omega$, the same as when the rotation was 640 turns.

We would like to study the longitudinal microwave instability limit for each time-advance slice of the beam. Here we wish to study a slice at time-advance $\tau=5.5 \mathrm{~ns}$, which is shown by the blue vertical line in Fig. 1. This line intersects the beam in two energy-offset-disconnected regions, the one close to the core has mean energy offset of $\Delta E_{1}=-0.00571$ while the one near the tail has mean energy offset of $\Delta E_{2}=0.0133$. We designate the region near the core 


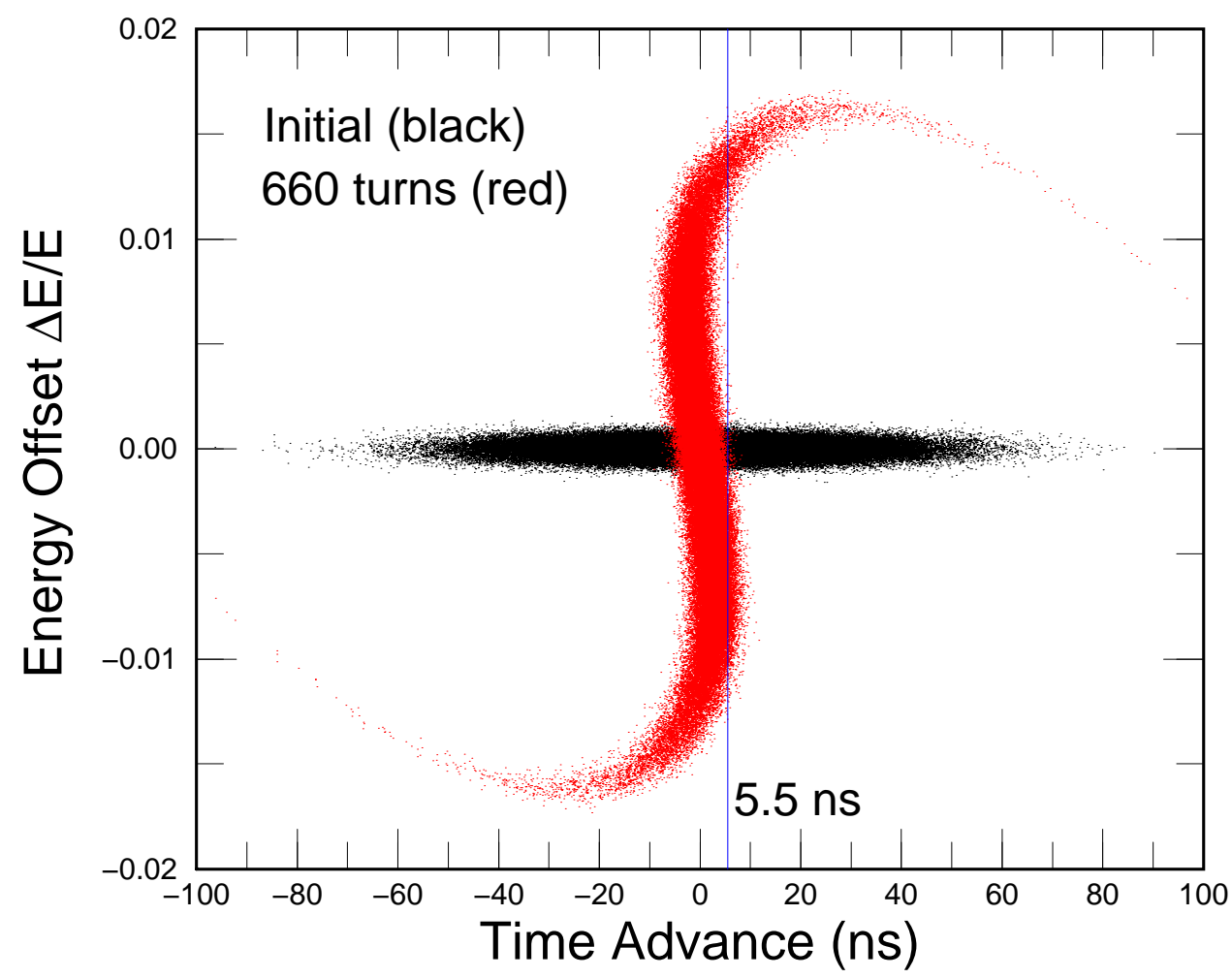

Figure 3: (Color) A bunch in the CR initially bi-Gaussian distributed with $\sigma_{\tau 0}=29.2 \mathrm{~ns}$ and $\sigma_{E 0}=5.2 \times 10^{-4}$ is shown in black dots. It is rotated by a $2.5-\mathrm{MHz} \mathrm{rf}$ of peak voltage $240 \mathrm{kV}$. Red dots show the bunch rotation after 660 turns in order to include more particles in the later truncated narrow bunch. Small-amplitude synchrotron period is 2403 turns. The vertical blue line is at $5.5 \mathrm{~ns}$ cut the beam at two regions that are disconnected in energy offset. .

as region 1 and the one near the tail as region 2. Again we sample particles within the slice $5.5 \pm 0.7$ ns. There are 3210 macroparticles in region 1 and 861 macroparticles in region 2. The fractional energy spread in the two regions are, respectively, $\sigma_{E 1}=2.64 \times 10^{-3}$ and $\sigma_{E 2}=1.15 \times 10^{-3}$. The deviation and fractional spread in energy lead to the deviation and spread in angular revolution frequency according to

$$
\Delta \omega_{0 i}=-\frac{\eta \bar{\omega}_{0}}{\beta^{2}} \Delta E_{i}
$$

and

$$
\sigma_{\omega_{0 i}}=\frac{|\eta| \bar{\omega}_{0}}{\beta^{2}} \sigma_{E i}
$$

where $\bar{\omega}_{0}$ is the nominal angular revolution frequency of the whole beam. These numbers are summarized and listed in Table I. We need to answer the question, what energy spread 
we should insert into the Boussard-modified Keil-Schnell criterion for the time-advance slice at $5.5 \mathrm{~ns}$ ?

Table I: Properties of the two disconnected regions of the 5.5-ns time-advance slice.

\begin{tabular}{lrr}
\hline & Region 1 & Region 2 \\
\hline Slice fraction $\alpha_{i}$ & 0.887 & 0.113 \\
Mean energy offset $\Delta E_{i}$ & $-5.71 \times 10^{-3}$ & $1.33 \times 10^{-2}$ \\
Rms energy spread $\sigma_{E i}$ & $2.64 \times 10^{-3}$ & $1.15 \times 10^{-3}$ \\
Mean angular rev. freq. $\Delta \omega_{0 i}$ & $-355 \mathrm{~s}^{-1}$ & $825 \mathrm{~s}^{-1}$ \\
Rms angular rev. freq. spread $\sigma_{\omega_{0 i}}$ & $165 \mathrm{~s}^{-1}$ & $71.3 \mathrm{~s}^{-1}$ \\
\hline
\end{tabular}

\subsection{Dispersion relation for overlapped beams}

The dispersion relation describing longitudinal microwave instability can be expressed as [4]

$$
1=-\left(\frac{\Delta \Omega_{0}}{n}\right)^{2} \int_{C} d \Delta \omega_{0} \frac{f^{\prime}\left(\Delta \omega_{0}\right)}{\frac{\Delta \Omega}{n}-\Delta \omega_{0}},
$$

where $\Delta \omega_{0}=\omega_{0}-\bar{\omega}_{0}$ is the angular revolution frequency of a particle relative to the mean angular revolution frequency $\bar{\omega}_{0}$ of the whole beam, and $\Delta \Omega / n=\Omega / n-\bar{\omega}_{0}$ with $\Omega$ being the collective frequency of the disturbance which drives the longitudinal microwave growth of the whole beam. The contour of integration $C$ runs below the pole $\Delta \omega_{0}=\Delta \Omega / n$. In the numerator of the integrand, $f\left(\Delta \omega_{0}\right)$ is the revolution frequency distribution of the slice of beam under investigation. Obviously, when there is no revolution frequency spread, $f\left(\Delta \omega_{0}\right)=\delta\left(\Delta \omega_{0}\right)$. Therefore $(\Delta \Omega)_{0}$ represents the shift of the $n^{\text {th }}$ harmonics of the angular revolution frequency driven by coupling impedance $Z_{0}^{\|}$of the vacuum chamber in the absence of Landau damping. In fact, it can be easily shown that

$$
\left(\frac{\Delta \Omega_{0}}{n}\right)^{2}=\frac{i e \eta \bar{\omega}_{0}^{2} I_{t}}{2 \pi \beta^{2} E} \frac{Z_{0}^{\|}}{n},
$$

where $I_{t}$ is the total local current at that time-advance slice including all the disconnected regions. Then the local current for the $i^{\text {th }}$ disconnected region will be $I_{i}=\alpha_{i} I_{t}$.

We assume that each of the $m$ disconnected regions are Gaussian distributed in angular revolution frequency with mean $\Delta \omega_{0 i}$ and rms spread $\sigma_{\omega_{0 i}}$. Then the revolution frequency 
distribution can be approximated as

$$
f\left(\Delta \omega_{0}\right)=\sum_{i=1}^{m} \frac{\alpha_{i}}{\sqrt{2 \pi} \sigma_{\omega_{0 i}}} e^{-\left(\Delta \omega_{0}-\Delta \omega_{0 i}\right)^{2} / 2 \sigma_{\omega_{0 i}}^{2}},
$$

where the $i^{\text {th }}$ region has mean angular revolution frequency $\Delta \omega_{0 i}$ and rms fractional angularrevolution-frequency spread $\sigma_{\omega_{0 i}}$. Just as for one connected region, this frequency distribution is normalized to unity when integrated over $\Delta \omega_{0}$. Thus $\sum_{i} \alpha_{i}=1$, where $\alpha_{i}$ is fraction of particles in this time-advance slice residing in the $i^{\text {th }}$ disconnected region. The integral in the dispersion relation of Eq.(3.24) can now be integrated in the closed form to give

$$
\left(\frac{\Delta \Omega_{0}}{n}\right)^{-2}=-\sum_{i}^{m} \frac{\alpha_{i}}{\sigma_{\omega_{0 i}}^{2}}\left[1+i \sqrt{\pi} u_{i} w\left(u_{i}\right)\right],
$$

where

$$
u_{i}=\frac{\Delta \Omega / n-\Delta \omega_{0 i}}{\sqrt{2} \sigma_{\omega_{0 i}}}
$$

and $w\left(u_{i}\right)$ is the complex error function. The stability contour can then we mapped out by letting $\Delta \Omega / n$ be real and vary it from $-\infty$ to $+\infty$. Before doing that let us extract the simplified stability limit or the so-call Boussard-modified Keil-Schnell limit. The method is to choose a particular value for $\operatorname{Re} \Delta \Omega / n$ so that the dispersion relation can be easily solved. For region $j$, this is the point when

$$
\mathcal{R e} u_{j}=\operatorname{Re} \frac{\Delta \Omega / n-\Delta \omega_{0 j}}{\sqrt{2} \sigma_{\omega_{0 j}}}=0
$$

Here, there are $m$ such points. For example, if we take the point $\mathcal{R} e u_{j}=0$, the term involving $i=j$ in Eq. (3.27) vanishes. But when $i \neq j$, we have

$$
u_{i}=\frac{\Delta \omega_{j}-\omega_{i}}{\sqrt{2} \sigma_{\omega_{0 i}}} \gg 1
$$

because of regions $i$ and $j$ are well separated. The complex error function can be expanded asymptotically using

$$
u_{i} w\left(u_{i}\right)=\frac{i}{\sqrt{\pi}}\left[1+\frac{1}{2 u_{i}^{2}}+\mathcal{O}\left(\frac{1}{\left|u_{i}\right|^{4}}\right)\right]
$$

Substitution into Eq. (3.27) gives

$$
\left(\frac{\Delta \Omega_{0}}{n}\right)^{-2}=-\frac{\alpha_{j}}{\sigma_{\omega_{0 j}}^{2}}+\sum_{i \neq j} \frac{\alpha_{i}}{\left(\Delta \omega_{j}-\Delta \omega_{i}\right)^{2}} .
$$


Since $\left(\Delta \omega_{j}-\Delta \omega_{i}\right)^{2} \gg \sigma_{\omega_{0 j}}^{2}$, if all the $\alpha_{i}$ 's are of comparable magnitude, all the terms in the summation for $i \neq j$ can be neglected, leaving behind the simple expression

$$
\left(\frac{\Delta \Omega_{0}}{n}\right)^{-2}=-\frac{\alpha_{j}}{\sigma_{\omega_{0 j}}^{2}} .
$$

We next make substitution from Eqs. (3.23) and (3.25) to arrive at

$$
-i \operatorname{sgn}(\eta) \frac{Z_{0}^{\|}}{n}=\frac{2 \pi|\eta| E}{e I_{i} \beta^{2}} \sigma_{E i}^{2},
$$

where $I_{i}=\alpha_{i} I_{t}$ is the local current of the $i^{\text {th }}$ region in the time-advance slice. This is just the Boussard-modified Keil-Schnell stability limit for the $i^{\text {th }}$ beam region of the time-advance slice, as if all other disconnected regions are absent. Below transition $(\eta<0)$, this limited impedance is inductive as speculated.

The above result can also be visualized as follows. Consider $m$ coasting beams, the $i^{\text {th }}$ one has mean revolution frequencies $\omega_{0}+\Delta \omega_{0 i}$ and rms spread $\sigma_{\omega_{0 i}}$. They are separated in the longitudinal phase space. Imagine a small perturbing current wave of the form $e^{i n \theta-i \Omega t}$, where $\theta$ is the azimuthal angle around the accelerator ring. If the coherent frequency is $\Omega \sim n\left(\omega_{0}+\Delta \omega_{i}\right)$, it will set the particles in the $i^{\text {th }}$ beam to oscillate near harmonic $n$ and eventually lead to a growth if $\sigma_{\omega_{0 i}}$ is not large enough to destroy the coherency. If $\sigma_{\omega_{0 i}} \ll\left|\Delta \omega_{0 j}-\omega_{0 i}\right|, i \neq j$, the particle in the $i^{\text {th }}$ beam will not be affected. Thus the stability criterion applies to each beam individually as if the others are not present.

Let us now apply the derivation to the two disconnected regions in the time-advance $\tau=5.5$-ns slice. The results are listed in Table II. From the table, we learn that the center slice of the beam has the most stringent stability limit. This is in agreement with the investigation using linear rf waveform.

\subsection{Stability Curve}

We let $\Delta \Omega$ have an infinitesimal positive imaginary part in the dispersion relation, Eq. (3.27). For each value of $\mathcal{R} e \Delta \Omega / n,\left(\Delta \Omega_{0} / n\right)^{2}$ is computed. The locus of $\left(\Delta \Omega_{0} / n\right)^{2}$ is the stability contour. Actually, we plot the stability contour in the complex $W$-plane, where

$$
W=-i \frac{\operatorname{sgn}(\eta)}{\sum_{i} \sigma_{\omega_{0 i}}^{2}}\left(\frac{\Delta \Omega_{0}}{n}\right)^{2} .
$$


Table II: Local currents and Keil-Schnell stability limits for disconnected regions of the 5.5-ns slice. Those for the 0 -ns slice after and before rotation are also shown for comparison.

\begin{tabular}{lcc}
\hline & $\begin{array}{c}\text { Local current } \\
I_{i}\end{array}$ & $\begin{array}{c}\text { Keil-Schnell Limit } \\
\left|Z_{0}^{\|} / n\right|\end{array}$ \\
\hline After rotation & & \\
5.5-ns slice core region & $130 \mathrm{~A}$ & $36.3 \Omega$ \\
5.5-ns slice tail region & $30.4 \mathrm{~A}$ & $27.1 \Omega$ \\
0-ns slice & $1145 \mathrm{~A}$ & $16.9 \Omega$ \\
Before rotation & & \\
0-ns slice & $115 \mathrm{~A}$ & $1.3 \Omega$ \\
\hline
\end{tabular}

The reason of such a choice is three-fold: it is dimensionless, it is directly proportional ${ }^{*}$ to $Z_{0}^{\|} / n$, and it is symmetric with respect to all the disconnected regions, (see Eq. (3.25)).

Our application is for the time-advance slice at $5.5 \mathrm{~ns}$, which consists of two disconnected regions: region 1 represents the core and region 2 represents the tail. All the inputs are taken from Table I. The stability contour for this time-advance slice is shown in red in Fig. 4. Three points of interest are marked by black dots. The $W$-space consists of two Riemann sheets with the positive $\operatorname{Im} W$-axis serving as the Riemann cut. The stability contour starts from the first quadrant of the $W$-space with $\operatorname{Re} \Delta \Omega /\left(n \sqrt{2} \sigma_{\omega_{01}}\right) \approx-4$.6. As $\operatorname{Re} \Delta \Omega /\left(n \sqrt{2} \sigma_{\omega_{01}}\right)$ increases, the contour progresses in the direction of the arrows wrapping around Point $A$, going upwards to reach Point B. It then crosses the Riemann cut and goes into the second Riemann sheet. There, it reverses direction at Point B going downwards and wraps around Point C. The first Riemann sheet is for the disconnected core region and Point A corresponds to the Keil-Schnell limit for that region. The second Riemann sheet is for the disconnected tail region and point B corresponds to the Keil-Schnell limit for that region. The stability contour clearly tells us that the stability limit for the tail region is larger than that in the core region. Numerically, these Keil-Schnell limits are listed in Table II.

We plot alongside in Fig. 4 in blue the stability contour of the time-advance slice at 0 ns, which consists of only one connected Gaussian distributed region. ${ }^{\dagger}$ It is well known

\footnotetext{
*Here, the proportionality constant is real and positive, so that the stability contour in the complex $Z_{0}^{\|} / n$-plane will look exactly the same.

${ }^{\dagger}$ The bell-bottom point of this blue curve is in between points $\mathrm{A}$ and $\mathrm{C}$. But this does not imply the
} 


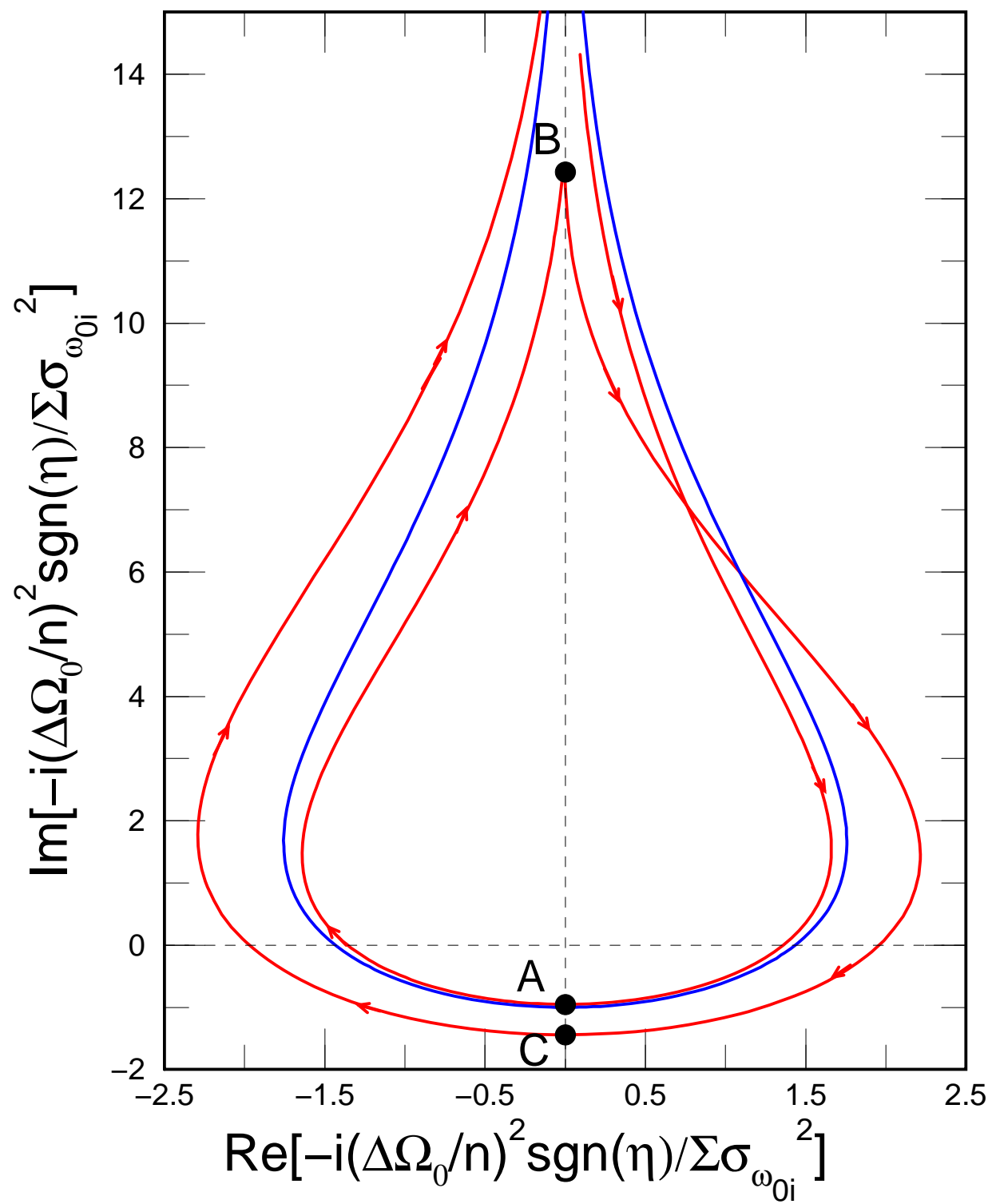

Figure 4: (Color) Red: Stability contour for the 5.5-ns time-advance slice in the complex $W$ plane, where $W$ is proportional to $Z_{0}^{\|} / n$ (see Eq. (3.35)). This time-advance slice consists of two disconnected regions: region 1 the core and region 2 the tail. Their properties are listed in Table I. Points A and C correspond, respectively, to the Keil-Schnell limits for the core region and the tail region. The positive $\mathcal{I} m W$-axis is a Riemann cut separating two Riemann sheets, one for the core region and one for the tail region. Blue: Stability contour for the time-advance slice at 0 ns, which consists of only one connected Gaussian distributed region. 
that the stability area enclosed by the stability contour can accommodate infinite capacitive $Z_{0}^{\|} / n$ provided that $\operatorname{Re} Z_{0}^{\|} / n=0$. This is quite different when there are disconnected regions in the time-advance slice. In our application, there is Point $\mathrm{C}$ on the Riemann cut, which separates the two Riemann sheets. The finite value of $\operatorname{Im} W$ (Point $\mathrm{C}$ ) indicates that only finite capacitive $Z_{0}^{\|} / n$ can be inside the stability contour. However, the difference may be of academic interest only, because Point $\mathrm{C}$ corresponds to $\operatorname{Im} W=12.4$ or a capacitive impedance of $Z_{0}^{\|} / n \approx i 430 \Omega$, which may be too big to stir any worry.

\section{Conclusions}

Intense proton bunches each of intensity $0.525 \times 10^{14}$ and rms length $29.2 \mathrm{~ns}$ are compressed to a narrow width of $\sigma_{\tau} \sim 3.2 \mathrm{~ns}$ in the $\mathrm{CR}$ by an rf voltage of $240 \mathrm{kV}$. We studied in this article the longitudinal microwave stability limit during the rf rotation. Nonlinear rf waveform leads to tail formation in the rotated beam. A time-advance slice may contain regions disconnected in energy offset. The stability limits of such disconnected regions were also investigated. Our analysis shows that as for longitudinal microwave instability, the beam will become more stable as the rf rotation progresses. The stability limit of the original prerotated lengthy beam of small energy spread will be studied in another article.

\section{References}

[1] E. Keil and W. Schnell, CERN Report TH-RF/69-48 (1969); E. Keil and A.M. Sessler, Rev. Sci. Instrum. 36, 429 (1965).

[2] D. Boussard, CERN LAB II/RF/Int./75-2 (1975).

[3] S. Krinsky and J.M. Wang, Particle Accelerators 17, 109 (1985).

[4] See, for example, K.Y. Ng, Physics of Intensity Dependent Beam Instabilities, World Scientific, 2006, Chapter 5.

Boussard-modifed Keil-Schnell limit of the 0 -ns slice is in between the stability limits for the disconnected core- and tail-regions of the 5.5-ns time-advance slice. This is because the local current $I_{t}$ and the energy spread $\sigma_{\omega_{0 i}}$ embedded in $W$ are very different for the two slices. 
[5] K.Y. Ng, AIP Conf. Proc. 150, Intersections Between Particle and Nuclear Physics, Lake Louism Canada, 1986, Ed. Donald F. Geesaman, New, York, p.401; K.Y. Ng, Microwave Instability Criterion for Overlapped Bunches, Fermilab Physics Note PN-432, 1986. 